\title{
CALCULATED TRAVEL TIMES OF SEISMIC CORE WAVES*
}

By R. D. Forester

\section{ABSTRACT}

Travel times for the seismic core waves, PKP, PKS, and SKS, were computed by integration along the travel paths. For this purpose the velocity distribution within the earth was broken into segments which were represented by continuous functions. Except for rays of grazing incidence to the outer core the times calculated for PKP and PKS are intermediate between the smoothed times given by Jeffreys and times based upon recent observed data. The times calculated for SKS are in fair agreement with the smoothed times given by Jeffreys.

\section{INTRODUCTION}

IN ORDER to estimate the intensity of energy flux expected for seismic waves at various epicentral distances along the earth's surface, travel times must be known with a high degree of precision. This is especially true near a focal point, where the slope of the travel-time curve changes rapidly. Observed travel-time data are frequently not precise enough for a determination of the curvature of the travel-time

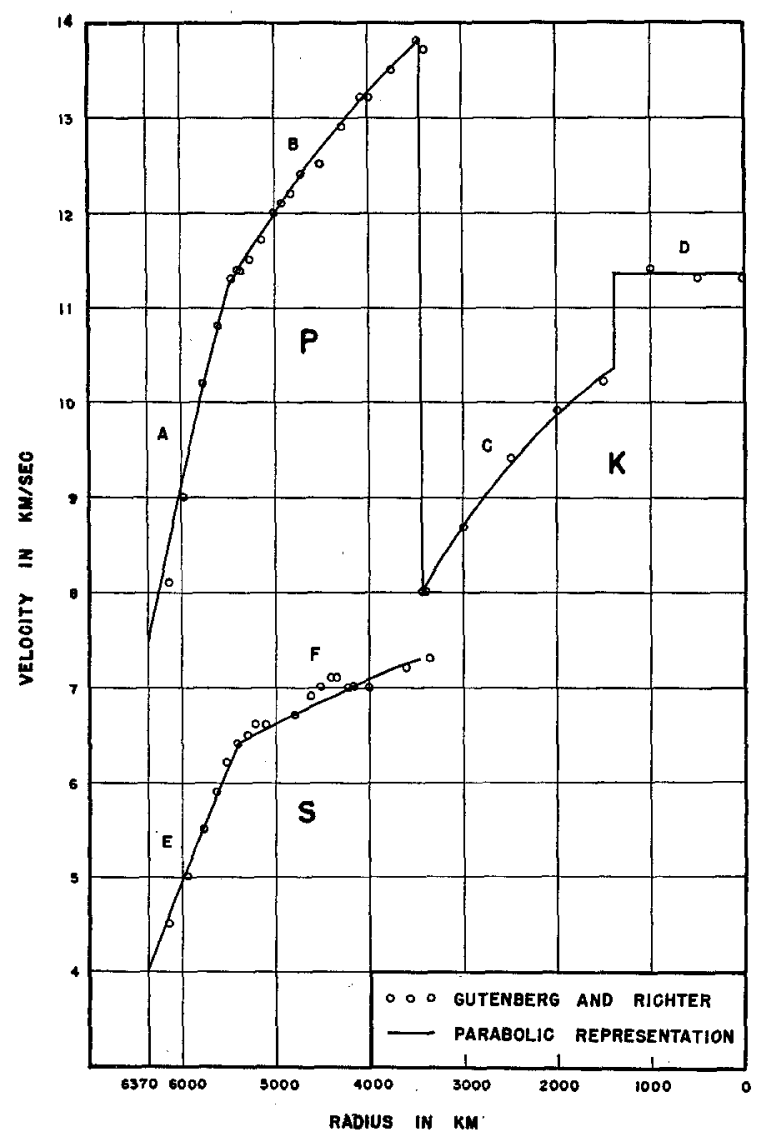

Fig. 1.

* Manuscript received for publication August 3, 1954. 
curves, a factor which is crucial in the analysis of the energy content of seismic waves. It was this fact which prompted the writer in his study of the energy content of the seismic core wave PKS to devise a method for computing its travel times. Travel times for PKP and SKS were computed as an additional check on the effectiveness of the computational method.

\section{Computational Method}

The travel times were computed directly by integration along the travel paths. Use was made of velocities for the mantle and the core given by Gutenberg and Richter. (See fig. 1.) These velocities are listed, respectively, in tables 69 and 73 of Gutenberg $(1951 b)$. The large magnitude of the gradients of these velocities indicates that large errors would result if constant velocities, and hence straight ray paths, were as-

TABLE 1

Boundary Condtions for the Calculation of the Travel-Time Curves

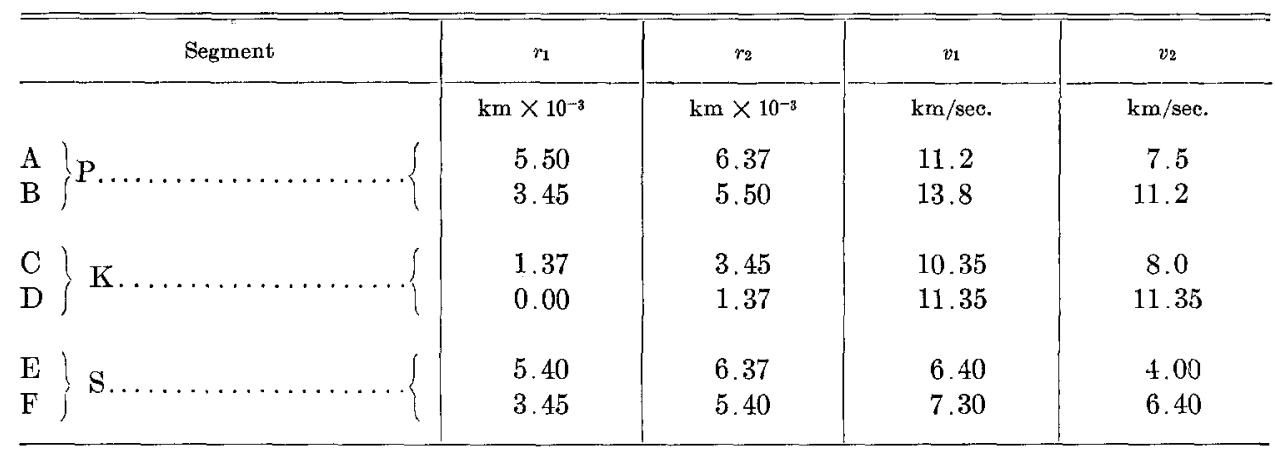

sumed for transverse waves in the mantle, and for longitudinal waves in the mantle and in the core. Instead, the velocity curves were broken into six segments, designated by the letters A through F. Segments A and B (longitudinal velocities in the core) and segments $\mathrm{E}$ and $\mathrm{F}$ (transverse velocities in the mantle) were expressed by the parabolic function:

$$
V=V_{0}-K r^{2}
$$

where $r$ is the radius from the center of the earth, and $V_{0}$ and $K$ are constants. The ray paths corresponding to such a velocity function are arcs of circles.

Since the velocity discontinuity at the inner core boundary appears to be gradational, continuous travel times were computed for only those waves which travel through the outer core. The segment $\mathrm{D}$ (velocity of the inner core) was assumed to be constant, and was used to calculate the travel times of only those waves which travel diametrally through the earth. Segments A, B, and C were used to compute the travel times of PKP; segments E, F, and C, those of SKS; and segments A, B, $\mathrm{C}, \mathrm{E}$, and $\mathrm{F}$, those of PKS.

The boundary conditions imposed on equation 1 in order to evaluate the parameters $V_{0}$ and $K$ are shown in table 1 . As shown in figure 1 , the agreement between the parabolic representation of the velocities and the data of Gutenberg and Richter is close.

If the boundary conditions in table 1 are substituted into equation (1), two simultaneous linear equations result which can be solved for $V_{0}$ and $K$ : 


$$
\begin{gathered}
V_{0}=\frac{v_{1} r_{2}^{2}-v_{2} r_{1}^{2}}{r_{2}^{2}-r_{1}^{2}} \\
K=\frac{v_{1}-v_{2}}{r_{2}^{2}-r_{1}^{2}}
\end{gathered}
$$

Where $r_{1}=$ inner radius bounding a ray-path segment

$r_{2}=$ outer radius bounding a ray-path segment

$v_{1}=$ velocity where inner radius bounds a ray-path segment

$v_{2}=$ velocity where outer radius bounds a ray-path segment.

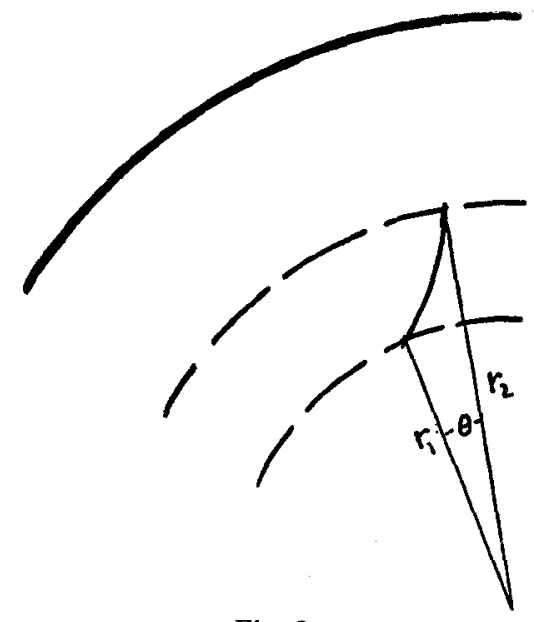

Fig. 2

By the application of Fermat's principle of stationary time, it can be shown that the central angle subtended by a ray-path segment for the velocity function defined by equation (1) is:

$$
\begin{aligned}
\theta=\frac{1}{2}\left\{\arcsin \left[\frac{1+2 p^{2} V_{0} K-2 p^{2} K^{2} r_{2}^{2}}{\sqrt{1+4 p^{2} V_{0} K}}\right]+\arcsin \left[\frac{1+2 p^{2} V_{0} K-\frac{2 p^{2} V_{0}^{2}}{r_{2}^{2}}}{\sqrt{1+4 p^{2} V_{0} K}}\right]\right. \\
\left.-\arcsin \left[\frac{1+2 p^{2} V_{0} K-2 p^{2} K^{2} r_{1}^{2}}{\sqrt{1+4 p^{2} V_{0} K}}\right]-\arcsin \left[\frac{1+2 p^{2} V_{0} K-\frac{2 p^{2} V_{0}^{2}}{r_{1}^{2}}}{\sqrt{1+4 p^{2} V_{0} K}}\right]\right\}
\end{aligned}
$$

where " $p$ " is equal to the ray parameter. Similarly, it can be shown that the corresponding time of travel along a ray path segment is:

$$
t=\frac{1}{2 \sqrt{V_{0} K}} \log _{10}\left|\frac{1-\frac{v_{2}}{2 V_{0}}+\sqrt{1-\frac{v_{2}}{V_{0}}-p^{2} \frac{v_{2}^{2}}{V_{0}} K}}{1-\frac{v_{1}}{2 V_{0}}+\sqrt{1-\frac{v_{1}}{V_{0}}-p^{2} \frac{v_{1} V_{0}}{V_{0} K}}} \times \frac{v_{1}}{v_{2}}\right|
$$

Values for $V_{0}$ and $K$ can be evaluated from equations (2) and (3), respectively. If the ray path does not penetrate as deep as the inner boundary radius, $r_{1}$, the expression for the central angle reduces to: 
TABLE 2

Calculated Travel Tmes

\begin{tabular}{|c|c|c|c|c|c|c|}
\hline $1 / V$ & $\stackrel{\Delta}{\mathrm{PKP}}$ & $\begin{array}{l}t \\
\mathrm{PKP}\end{array}$ & $\mathrm{P} \overrightarrow{\mathrm{KS}}$ & $\stackrel{t}{\text { PKS }}$ & sKs & $\begin{array}{l}t \\
\text { sKs }\end{array}$ \\
\hline $\mathrm{sec} / \mathrm{deg}$. & deg. & sec. & deg. & sec. & deg. & see. \\
\hline 0.000 & 180.000 & 1201.06 & 180.000 & 1416.09 & 180.000 & 1631.13 \\
\hline 2.310 & 149.345 & 1186.60 & 142.776 & 1393.77 & 136.207 & 1600.94 \\
\hline 2.319 & 149.264 & 1186.41 & 142.663 & 1393.50 & 136.061 & 1600.58 \\
\hline 2.383 & 148.688 & 1185.06 & 141.838 & 1391.56 & 134.988 & 1598.06 \\
\hline 2.446 & 148.136 & 1183.73 & 141.031 & 1389.61 & 133.926 & 1595.50 \\
\hline 2.510 & 147.610 & 1182.42 & 140.241 & 1387.66 & 132.873 & 1592.89 \\
\hline 2.574 & 147.109 & 1181.14 & 139.470 & 1385.69 & 131.831 & 1590.24 \\
\hline 2.638 & 146.637 & 1179.92 & 138.719 & 1383.74 & 130.801 & 1587.55 \\
\hline 2.701 & 146.193 & 1178.74 & 137.987 & 1381.78 & 129.780 & 1584.83 \\
\hline 2.765 & 145.781 & 1177.60 & 137.276 & 1379.83 & 128.771 & 1582.07 \\
\hline 2.828 & 145.400 & 1176.54 & 136.586 & 1377.91 & 127.772 & 1579.28 \\
\hline 2.892 & 145.052 & 1175.55 & 135.918 & 1376.00 & 126.784 & 1576.45 \\
\hline 2.955 & 144.740 & 1174.63 & 135.274 & 1374.11 & 125.808 & 1573.60 \\
\hline 3.018 & 144.466 & 1173.82 & 134.654 & 1372.27 & 124.841 & 1570.72 \\
\hline 3.082 & 144.231 & 1173.10 & 134.059 & 1370.45 & 123.886 & 1567.80 \\
\hline 3.145 & 144.040 & 1172.50 & 133.491 & 1368.68 & 122.942 & 1564.86 \\
\hline 3.208 & 143.894 & 1172.05 & 132.951 & 1366.97 & 122.009 & 1561.90 \\
\hline 3.271 & 143.797 & 1171.72 & 132.442 & 1365.31 & 121.087 & 1558.90 \\
\hline 3.334 & 143.757 & 1171.58 & 131.966 & 1363.74 & 120.176 & 1555.90 \\
\hline 3.397 & 143.765 & 1171.63 & 131.520 & 1362.25 & 119.275 & 1552.87 \\
\hline 3.460 & 143.841 & 1171.89 & 131.113 & 1360.85 & 118.385 & 1549.82 \\
\hline 3.523 & 143.985 & 1172.38 & 130.746 & 1359.56 & 117.507 & 1546.74 \\
\hline 3.586 & 144.205 & 1173.17 & 130.422 & 1358.41 & 116.638 & 1543.66 \\
\hline 3.649 & 144.510 & 1174.28 & 130.146 & 1357.42 & 115.781 & 1540.56 \\
\hline 3.711 & 144.913 & 1175.76 & 129.924 & 1356.59 & 114.934 & 1537.42 \\
\hline 3.774 & 145.426 & 1177.68 & 129.762 & 1356.00 & 114.098 & 1534.31 \\
\hline 3.836 & 146.067 & 1180.10 & 129.669 & 1355.62 & 113.272 & 1531.15 \\
\hline 3.899 & 146.859 & 1183.18 & 129.658 & 1355.60 & 112.457 & 1528.01 \\
\hline 3.961 & 147.834 & 1187.02 & 129.743 & 1355.93 & 111.651 & 1524.85 \\
\hline 4.023 & 149.037 & 1191.82 & 129.947 & 1356.75 & 110.856 & 1521.68 \\
\hline 4.086 & 150.535 & 1197.89 & 1.30 .303 & 1358.20 & 110.071 & 1518.50 \\
\hline 4.148 & 152.434 & 1205.72 & 130.866 & 1360.52 & 109.298 & 1515.31 \\
\hline 4.210 & 154.932 & 1216.16 & 131.734 & 1364.14 & 108.536 & 1512.11 \\
\hline 4.272 & 158.459 & 1231.12 & 133.119 & 1370.00 & 107.780 & 1508.91 \\
\hline 4.334 & 164.504 & 1257.29 & 135.769 & 1381.49 & 107.034 & 1505.69 \\
\hline 4.363 & 174.199 & 1299.38 & 140.442 & 1401.79 & 106.686 & 1504.20 \\
\hline
\end{tabular}


$\theta=\frac{1}{2}\left\{\arcsin \left[\frac{1+2 p^{2} V_{0} K-2 p^{2} K r_{2}^{2}}{\sqrt{1+4 p^{2} V_{0} K}}\right]+\arcsin \left|\frac{1+2 p^{2} V_{0} K-\frac{2 p^{2} V_{0}^{2}}{r_{2}^{2}}}{\sqrt{1+4 p^{2} V_{0} K}}\right|\right\}$

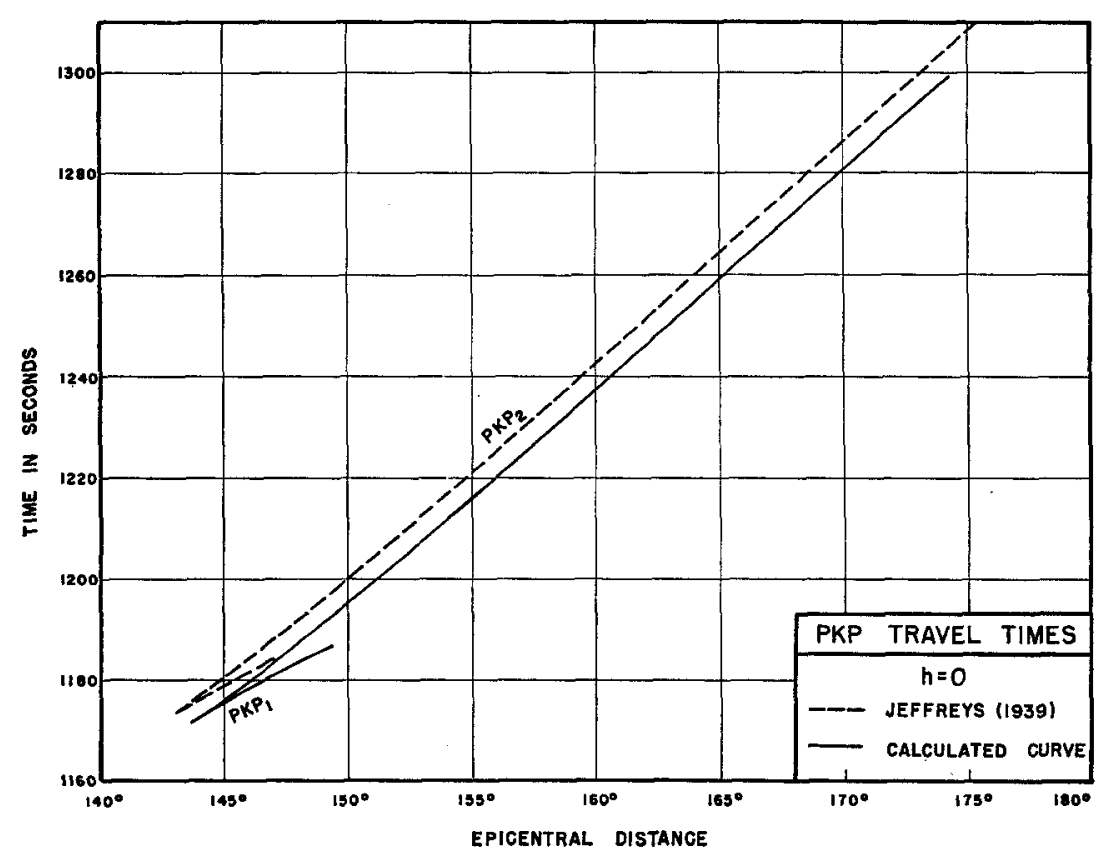

Fig. 3.

whereas the expression for the time becomes more complicated:

$$
t=\frac{1}{2 \sqrt{V_{0} K}} \log _{10}\left|\frac{2 \frac{V_{0}}{v_{2}}-1+2 \sqrt{\frac{V_{0}^{2}}{v_{2}^{2}}-p^{2} V_{0} K-\frac{V_{0}}{v_{2}}}}{\frac{4 p^{2} V_{0} K}{\sqrt{1+4 p^{2} V_{0} K}-1}-1}\right|
$$

The travel times and the central angles of the component segments of the core phases were computed from equations (4), (5), (6), and (7). These travel times and central angles were then combined, respectively, to obtain the total travel time as a function of the epicentral distance. In table 2, the resultant travel times for the core phases are listed as a function of the ray parameter. The ray parameter is equal to the reciprocal of the apparent velocity of a wave front as it emerges at the earth's surface, and hence is equal to the slope of the travel-time curve, $d t / d \Delta$, at the corresponding epicentral distance.

\section{Results}

In figures 3, 4, and 5, the calculated travel times can be compared with the smoothed travel times given by Jeffreys (1939b). The calculated times for PKP are several 


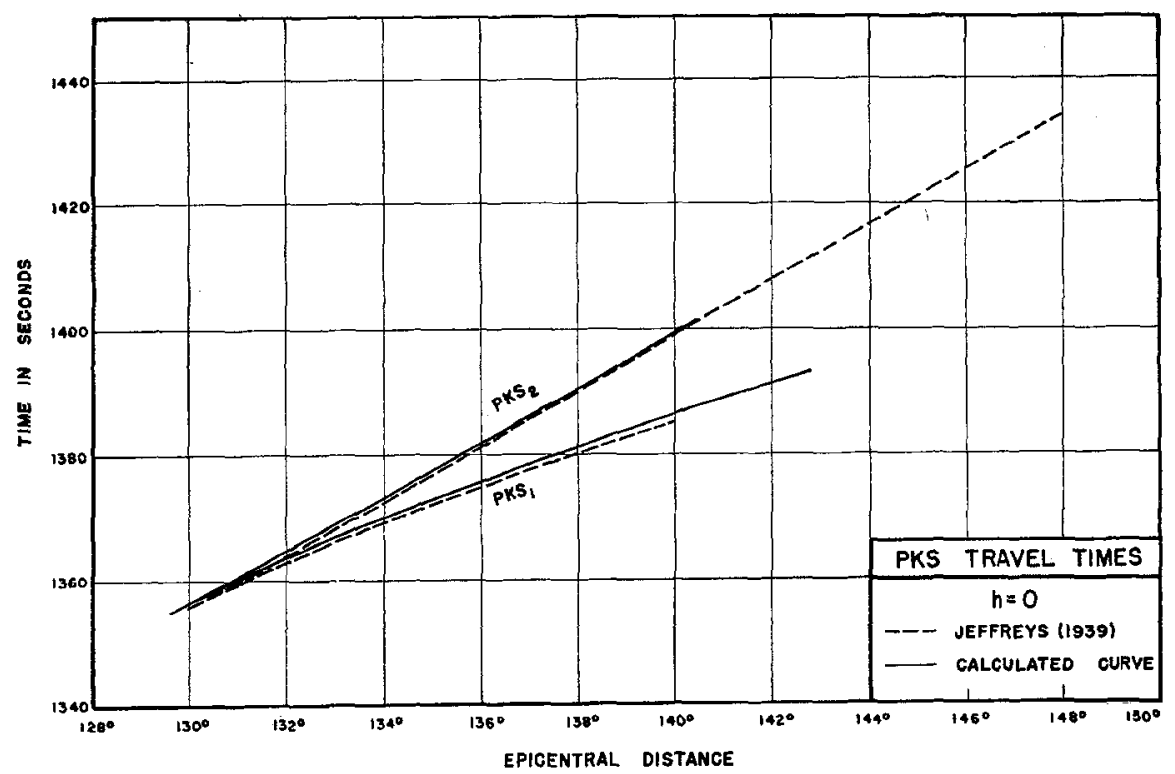

Fig. 4.

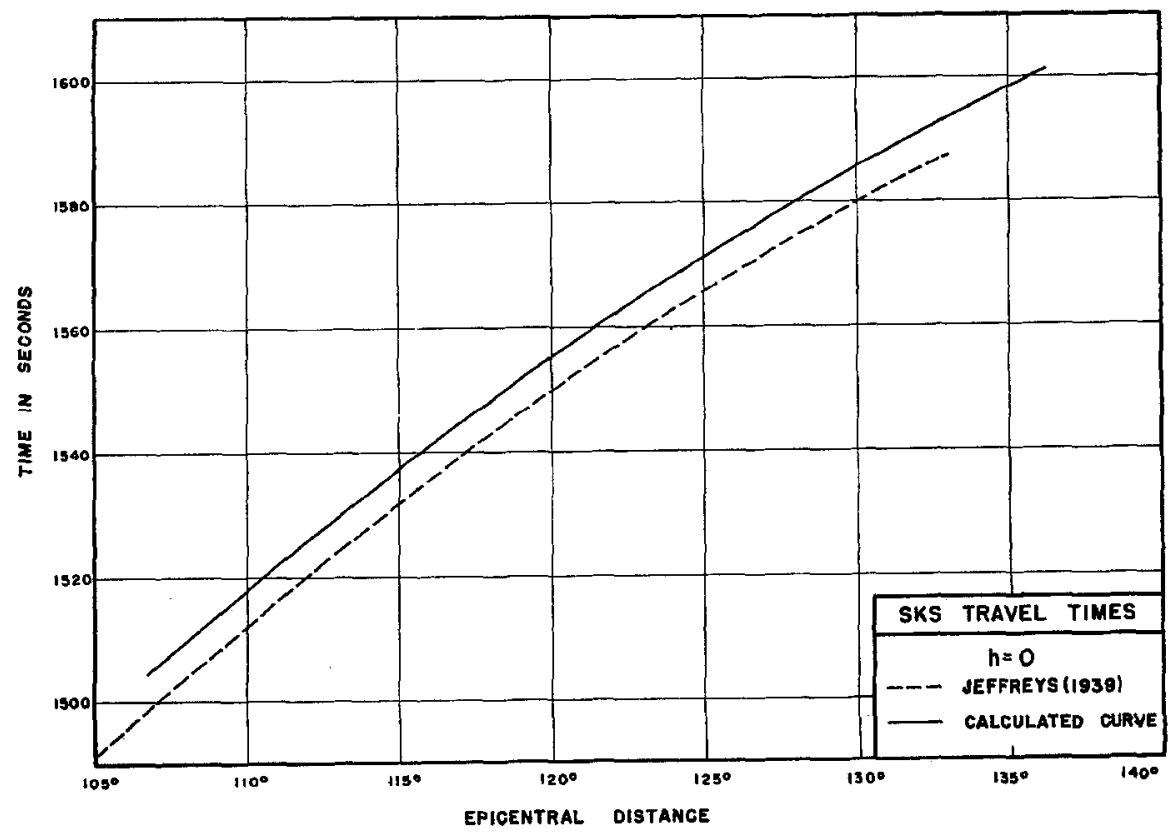

Fig. 5 .

seconds later than the smoothed times of Jeffreys; those for PKS, about equal to those of Jeffreys; and those for SKS, several seconds earlier than those of Jeffreys. Actually, the calculated times for PKP, PKS, and SKS would all be expected to be a few seconds earlier than the smoothed times of Jeffreys because the calculated 
times were computed from velocity functions for the mantle which did not incorporate the low velocities which prevail in the crust.

The paths computed for rays which graze the core are highly sensitive to the velocity assumed to exist outside the core boundary. The function used to represent the longitudinal velocity near the core did not follow the sharp decrease in velocity

TABLE 3

Travei-Time Comparisons of Core Phases

\begin{tabular}{|c|c|c|c|c|}
\hline \multirow[b]{2}{*}{ Phase } & \multicolumn{2}{|c|}{$\begin{array}{l}\text { Smoothed travel times of } \\
\text { Jeffreys (1939) }\end{array}$} & \multicolumn{2}{|c|}{ Calculated travel times } \\
\hline & $\begin{array}{l}\text { Epicentral } \\
\text { distance }\end{array}$ & Time & $\begin{array}{l}\text { Epicentral } \\
\text { distance }\end{array}$ & Time \\
\hline & deg. & sec. & deg. & sec. \\
\hline PKP through center of earth. & 180 & 1212.2 & 180 & 1201.1 \\
\hline PKS through center of earth.......... & 180 & 1422.9 & 180 & 1416.1 \\
\hline SKS through center of earth... & 180 & 1633.5 & 180 & 1631.1 \\
\hline PKP focus $\ldots \ldots \ldots \ldots \ldots \ldots \ldots$ & 143 & 1173.5 & 143.8 & 1171.6 \\
\hline PKS focus. . . . . . . . . . . . & 130 & 1356.2 & 129.6 & 1355.6 \\
\hline PKP grazing outer core $\ldots \ldots \ldots \ldots \ldots \ldots$ & 180 & 1330.6 & 174.2 & 1299.4 \\
\hline PKS grazing outer core $\ldots \ldots \ldots \ldots \ldots \ldots \ldots$ & 148 & 1435.0 & 140.4 & 1401.8 \\
\hline SKS maximum angle of incidence to outer core... & 62 & 1213.7 & 63.7 & 1232.3 \\
\hline PKP grazing inner core $\ldots \ldots \ldots \ldots \ldots \ldots \ldots \ldots$ & 147 & 1184.1 & 149.3 & 1186.6 \\
\hline PKS grazing inner core $\ldots . \ldots \ldots \ldots$ & 140 & 1385.7 & 142.8 & 1393.8 \\
\hline SKS grazing inner core $\ldots \ldots \ldots \ldots \ldots \ldots \ldots$ & 133 & 1587.3 & 136.2 & 1600.9 \\
\hline
\end{tabular}

gradient near the core boundary indicated by Gutenberg and Richter. (See fig. 1.) This may account for the fact that the distances computed for the PKP and PKS rays which graze the outer core were too small.

Listed in table 3 are some key comparisons between the calculated times and the smoothed times of Jeffreys. The focal point data for PKP and PKS are in excellent agreement. The largest discrepancies occur for the waves which graze the outer core.

The epicentral distance of the $\mathrm{PKP}_{2}$ ray which grazes the outer core was computed as $174^{\circ} .2$. Jeffreys gives a figure of $180^{\circ}$ for this distance. Jeffreys' figure is more valid because Gutenberg and Richter (1934) have observed $\mathrm{PKP}_{2}$ at a maximum distance of $180^{\circ}$.

The distance of the $\mathrm{PKS}_{2}$ ray which grazes the outer core was computed as $140^{\circ} 4$. Jeffreys gives a figure of $148^{\circ}$ for this distance. Neither figure compares favorably with a distance of $143^{\circ}$ predicted from a travel-time curve (hereafter designated as the "synthetic" PKS travel-time curve) synthesized by the method of Wadati and Masuda (1934) from travel times given by Jeffreys (1939a) for PcS and from travel times given by Gutenberg (1951a) for K.

For PKP and SKS, the slopes of the calculated curves match those of Jeffreys rather well; for PKS, they diverge noticeably. For the rays which penetrate close to the inner core, the slopes of the calculated curves would be expected to be significantly larger than those of the curves of Jeffreys, because Jeffreys (1952) calculates higher velocities for longitudinal waves in the outer core than does Gutenberg (1951a). (See fig. 6.) 
For PKP, the calculated curve agrees better with the observed data of Denson (1952) than does the curve of Jeffreys. The focal pint for the calculated curve occurs at $143 \% 8$, and that for the curve of Jeffreys at $143^{\circ}$. Denson, from a consideration of amplitude data, concludes that the focal distance for long-period waves occurs at $143^{\circ}$, and that for short-period waves at $147^{\circ}$. The distance of the $\mathrm{PKP}_{1}$

DEPTH IN KM

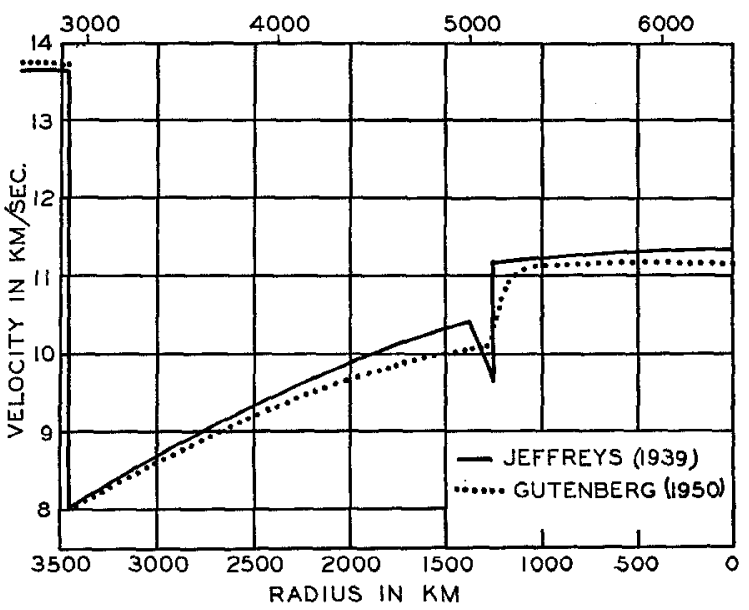

Fig. 6. Calculated velocity of longitudinal waves in the core.

ray which grazes the inner core is $149^{\circ} .3$ for the calculated curve, and $147^{\circ}$ for the curve of Jeffreys. Denson believes this distance to be about $157^{\circ}$.

For PKS, the main focal branch of the calculated curve, $\mathrm{PKS}_{1}$, agrees somewhat better with the observed data than does the curve of Jeffreys. The focal point of PKS occurs at $129^{\circ} 6$ for the calculated curve and at $130^{\circ}$ for the curve of Jeffreys. Forester (1953) has observed that the focal point occurs at $1311 / 2$ for waves with periods of 1 to 5 seconds, and between 130 and $131^{\circ}$ for waves with periods of 5 to 10 seconds.

The epicentral distance of the $\mathrm{PKS}_{1}$ ray which grazes the inner core is $142: 8$ for the calculated curve, $140^{\circ}$ for the curve of Jeffreys, and $149^{\circ}$ for the synthetic PKS curve. Long-period PKS waves have been observed with certainty by Forester (1953) at a maximum distance of $145^{\circ}$.

The epicentral distances at which the $P K P_{1}$ and $\mathrm{PKS}_{1}$ rays graze the inner core is less for the calculated curves than for the curves of Jeffreys, partly because Jeffreys has calculated $130 \mathrm{~km}$. less depth to the inner core than has Gutenberg; and partly because Jeffreys has calculated core velocities which have higher gradients than has Gutenberg. (See fig. 6.) High-velocity gradients produce greater ray curvature, and accordingly cause the central angle subtended by a ray in the core to be reduced.

The slopes of $\mathrm{PKS}_{1}$ for the calculated curve are higher than those for the curve of Jeffreys and lower than those for the synthetic PKS curve. The slopes of $\mathrm{PKS}_{2}$ are lower for the calculated curve than those for either the curve of Jeffreys or the synthetic curve. Forester has observed slopes for $\mathrm{PKS}_{1}$ more in accord with the calculated curve than the curve of Jeffreys. 


\section{CONCLUSIONS}

Travel times which are calculated by approximating the velocity distribution within the earth by an assemblage of continuous functions provide a basis for the unification and comparison of different types of seismic waves with each other. Such travel times can be calculated with a precision not attainable from observed data, and hence may be used as smoothed travel times where the observed data are crude, or as predicted travel times where observed data are lacking.

Travel times for rays which graze a discontinuity are difficult to calculate, for such rays are highly sensitive to the velocity proximal to the discontinuity.

Calculated travel times should aid in the attempt to correlate multiples of different seismic phases with each other, to analyze dispersion, and to detect where seismic ray paths might deviate markedly from the ray paths defined by Fermat's principle of stationary time.

To be of value, calculated travel times must be not only precise, but accurate. They are not a substitute for observed data. Wherever possible, they should be systematically adjusted to agree with the observed data.

\section{ReFerences}

Denson, M. E., JR.

1952. "Longitudinal Waves through the Earth's Core," Bull. Seism. Soc. Am., 42:119-134.

Forester, R. D.

1953. "Studies of the Travel Times, Periods, and Energy of Seismic Waves SKP and Related Phases," unpublished doctoral dissertation, California Institute of Technology, Pasadena, California.

GUTENBERg, B.

1951a. "PKKP, $\mathrm{P}^{\prime} \mathrm{P}$ ', and the Earth's Core," Trans. Am. Geophys. Union, 32: 373-390.

1951b. Internal Constitution of the Earth (Dover Pub.).

Gutenberg, B., and C. F. Richter

1934. "On Seismic Waves (First Paper)," Gerlands Beitr. z. Geophysik, Vol. 5, pp. 56-133.

JEFFREYS, H.

1939a. "The Time of PcP and SeS," Mon. Not. Roy. A stron. Soc., Geophys. Suppl., 4: 337-547.

1939b. "The Times of Core Waves (Second Paper)," ibid., 4: 594-615.

1952. The Earth (Cambridge Univ. Press), p. 115.

WADATI, K., and K. MASUDA

1934. "On the Travel Time of Earthquake Waves, Part VI," Geophys. Mag., Tokyo, 8: 187194.

California Institute of Technology

Pasadena, California

(Division of the Geological Sciences, contribution no. 689) 